\title{
LA ZONA CENTRO ORIENTE, UNA MIRADA DESDE LA COORDINACIÓN ZONAL DE INVESTIGACIÓN
}

\author{
Eliécer Pineda Ballesteros ${ }^{114}$
}

Recientemente se celebraron los 228 años de la insurrección comunera liderada por gente de esta bravía tierra. Entre ellos se destacaron José Antonio Galán y Manuela Beltrán quienes se manifestaban contrarios al mandato déspota de la corona española. Este artículo pretende ser una especie de homenaje al pueblo del gran Santander que ha demostrado su tesón y carácter fuerte a través de la historia, forma de ser que posibilitó ostentar un liderazgo visible en los procesos económicos, sociales, culturales, y otros, ocurridos en nuestra patria.

La zona centro oriente se encuentra conformada por cinco regiones geográficas bien definidas, lideradas por las municipalidades de Bucaramanga, Málaga, Vélez, Ocaña y Pamplona.

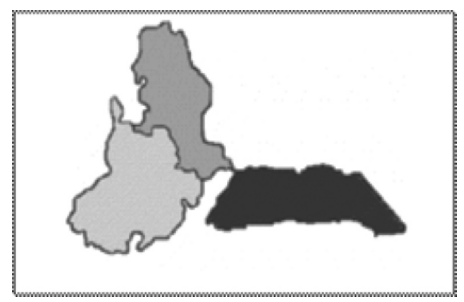

Figura 1. Zona Centro Oriente

\section{LA REGIÓN DE BUCARAMANGA}

La Parroquia de Nuestra Señora de Chiquinquirá y San Laureano del Real de Minas de Bucaramanga, según se puede leer en la página oficial de la alcaldía de Bucaramanga, fue el nombre que le otorgaron los habitantes a la parroquia fundada en el año de 1772, en el mismo lugar donde se hallaba el pueblo de indios «Real de Minas de Bucaramanga» establecido en el año de 1622 para la explotación del oro.

Con respecto de la palabra «Bucaramanga», se presume que tiene una raíz indígena aún cuando no haya estudios lingüísticos que aporten evidencia de ello. En 1857, la Villa de Bucaramanga es designada como capital del estado independiente de Santander, título que perdería cuatro años después con la Villa del Socorro, hasta que la Constitución de 1896 la declarara, definitivamente, como ciudad capital del departamento de Santander.

Consecuencia de la guerra de los mil días a principios del siglo xx, Bucaramanga padeció una crisis económica, pero en la segunda década del siglo xx comenzó su recuperación, mostró un gran crecimiento desde el punto de vista poblacional y de infraestructura. Hoy, Bucaramanga

114 Coordinador de investigaciones, Zona Centro Oriente. Correo electrónico: <eliecer.pineda@unad.edu.co>. 
es una de las cinco ciudades más importantes de Colombia, cuenta con cerca de un millón de habitantes en su área metropolitana y se caracteriza por contar con más de diez universidades, entre ellas la UNAD; tiene además, a disposición de los visitantes, un aeropuerto internacional, una significativa infraestructura hotelera acompañada por atractivos turísticos; todo ello, recreado a partir de la conocida hospitalidad del bumangués que le ha merecido el apelativo de «La Ciudad Bonita».

La ciudad es básicamente comercial aunque existen otros renglones representativos tales como la industria del calzado, la confección, la prestación de servicios de salud, finanzas y educación. Para el siglo xxi Bucaramanga se concibe a sí misma como la Tecnópolis de los Andes y prueba de ello es el creciente número de centros de investigación en diversos tópicos, como la energía, el gas, el petróleo, la corrosión, los asfaltos, el cuero y las herramientas de desarrollo agroindustrial, entre otros. También es un renglón importante en la economía, el agropecuario, cuyas principales actividades son la agricultura, la ganadería y la avicultura que se llevan a cabo en zonas aledañas de los departamentos de Santander y Cesar, pero su administración y comercialización se desarrollan en la ciudad.

En Bucaramanga se cuenta con un movimiento cultural que comienza a hacerse fuerte a nivel nacional. La pintura y la escultura tienen nuevos espacios para su difusión y el arte ha dejado de ser exclusivo para la élite, convirtiéndose paulatinamente en una parte integral de la ciudad. Exposiciones, retrospectivas, salones artísticos, conversatorios y talleres son una realidad en Bucaramanga. El festival de piano de la UIS, el festival iberoamericano de cuenteros, Santander en escena y el festivalito ruitoqueño son algunos de los eventos que anualmente se realizan en la ciudad y la llevan, paulatinamente, a ser uno de los centros culturales nacientes del país.

En el campo de la gastronomía son famosas las hormigas culonas, ${ }^{115}$ originarias de Santander y de la región que coincide con la antigua circunscripción de gran parte del imperio guane. Los guanes fueron un grupo indígena chibcha que habitó la región que actualmente ocupa gran parte de las provincias de Soto, Guanentá y Vélez, apreciaban tanto las culonas que las guardaban en calabazos para hacer provisión para muchos meses. Dentro del programa alimenticio de los guanes las hormigas culonas ocupaban un puesto básico. Se le considera un alimento afrodisíaco utilizado por los ancestros santandereanos en sus ceremonias nupciales, para llenar de vitalidad y larga vida a las parejas que respiraban su aroma.

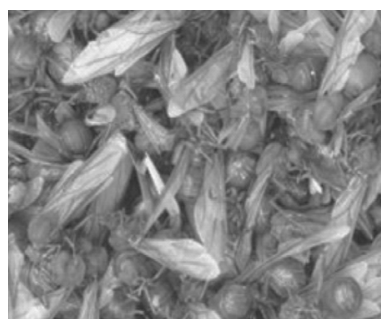

Figura 2. Hormigas culonas

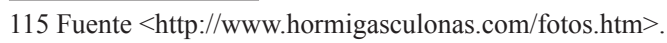


Finalmente, una de las atracciones cercanas a Bucaramanga es el parque Panachi, ${ }^{116}$ localizado a sólo $54 \mathrm{~km}$ de esta ciudad. El parque se ha convertido en el ícono que simboliza la pujanza y fortaleza del pueblo santandereano. Está ubicado en el cañón del Chicamocha, majestuoso e imponente que roba las miradas de todos los que se acercan a su territorio. La visita al parque se ha convertido en una actividad obligada de los turistas que llegan a Santander, es un encuentro con la naturaleza para poder tocar el cielo con las manos.

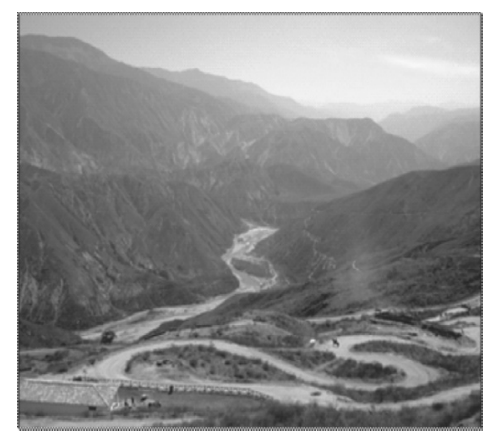

Figura 3. Cañón del Chicamocha visto desde el parque Panachi

\section{CEAD DE BUCARAMANGA}

Respondiendo al principio de «la educación para todos» la Unisur creó mediante acuerdo 028 de junio 21 de 1984, la Unidad Operativa de Educación Superior Abierta y a Distancia de Zapatoca. Cuatro años después la mayoría de estudiantes que presentaban matrícula vigente en la unidad operativa de Zapatoca, era oriunda de allí y de los municipios vecinos, pero también existía un número considerable procedente de Bucaramanga y su área metropolitana lo que permitió a las directivas de la Universidad adelantar acciones encaminadas a efectuar el traslado de dicho Centro a la Villa de los Caballeros de San Juan de Girón, mediante el acuerdo del Consejo Superior 018 del 25 de febrero de 1988.

En 1994 el CREAD se traslada al Colegio Metropolitano del Sur, en el municipio de Floridablanca, donde permaneció durante cinco años.

El primero de junio de 1999, el CREAD inicia actividades académicas en una sede que alquila ubicada en la Calle 3420 - 68, logra independencia, autonomía y mayor comodidad y, a su vez, existe un mayor sentido de pertenencia.

Gracias al apoyo de la Dirección General de la Universidad, y las directivas del Centro Regional, hoy se cuenta con una sede moderna, dotada de tecnología que permite poco a poco dar los primeros pasos en las NITC, hasta llegar a convertirnos en el verdadero Campo virtual como se tiene previsto en el plan de desarrollo del CEAD.

116 Fuente: <http://www.skyscrapercity.com/showthread.php?t=439598> 
Actualmente el CEAD tiene alrededor de 1323 estudiantes matriculados en pregrado y en postgrado; respecto a los egresados, estos son alrededor de 1350 profesionales en las diferentes áreas del conocimiento.

De otra parte el CEAD de Bucaramanga cuenta con tres grupos de investigación, el grupo GUANE adscrito a la Escuela de Ciencias Básicas, Tecnología e Ingeniería, que actualmente está en proceso de clasificación por Colciencias; el grupo Yariguies de la Escuela de Ciencias Sociales, Artes y Humanidades y el grupo GIAUNAD perteneciente a la Escuela de Ciencias Básicas, Tecnología e Ingeniería. Los dos últimos grupos están en proceso de consolidación.

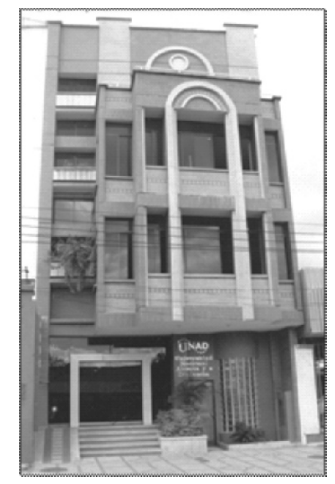

Figura 4. Panorámica del CEAD de Bucaramanga

En cuanto a semilleros se puede hacer el siguiente inventario: semillero Visionarios investiga en el campo del diseño de estrategias administrativas para las cadenas productivas; semillero Complexus trabaja en el campo de la neuropsicología y neurobiología del desarrollo; semillero Argumentación y Digalo cuyo objeto de estudio son los procesos de argumentación como apoyo en la comprensión de los problemas de familia; semillero Competir, Competencias Laborales que estudia la forma de realizar representaciones sociales sobre la violencia psicológica en las instituciones educativas; el semillero CHOGUA que reflexiona alrededor de los referentes teóricos desde los que se erige la psicología social, con miras a una mayor comprensión y apropiación de ellos; el semillero SIA que investiga acerca de la generación de nuevos procesos en la ingeniería de alimentos, y, el semillero ELEKTRON que busca indagar acerca de los fenómenos de tipo electrónico y su aplicación en la ingeniería.

\section{LA REGIÓN DE MÁLAGA}

La ciudad de Málaga se encuentra en la Provincia de García Roviraubicadaal nororiente del departamento de Santander, limítrofe con los departamentos de Boyacá y Norte de Santander, conformada por doce municipios que dependen administrativamente de la Gobernación del departamento.

La población es mayoritariamente de extracción campesina estratificada en los niveles 1 y 2 , de baja escolaridad, economía agraria de subsistencia, altamente heterogénea con una marcada presencia de mujeres jefe de hogar. Se presenta una notoria influencia de ingresos por mano de 
obra que en sectores supera los ingresos por productividad. Esta característica de inferioridad a la UAF (Unidad Agrícola Familiar) genera problemas de salubridad, migración e inconformismo social que sumen a la provincia en una crisis poblacional.

En materia de educación la población tiene fácil acceso al nivel primario, en un $60 \%$ al nivel de secundaria básica y en un mínimo porcentaje a la profesionalización o educación no formal. Todos los municipios cuentan con establecimientos de educación secundaria, pero regionalmente sólo se cuenta con la presencia de una universidad a nivel presencial, la UIS seccional Málaga, y universidades de modalidad a distancia entre las cuales se destaca la UNAD. Así mismo, juegan papel preponderante el SENA y el SAT.

Las características agroecológicas de la provincia de García Rovira, la perfilan como una región de actividades agrarias y en efecto sus suelos producen el 40\% del tabaco nacional. Anualmente la cosecha de papa se valora por encima de los doce mil millones de pesos, paralelamente con reglones como el ajo y la ganadería de leche y carne. La industrialización en García Rovira ha sufrido un notorio retroceso debido a variables como el aislamiento vial, la situación política y, en gran parte, por el contrabando, pues llevaron a que industrias como los molinos de trigo desaparecieran.

En lo que tiene que ver con los aspectos culturales, Málaga cuenta con el carnaval del Oriente Colombiano y las fiestas Patronales Municipales. En el campo de las artes hacen presencia en la zona los maestros en pintura Gustavo Gelvez, Hugo Moreno, Cayo Moreno y Carlos Meza. También tienen su sede de trabajo en la zona importantes escritores como la poetisa María Victoria Meneses, el poeta Álvaro Mancera y, respecto de la música, se distingue el grupo carranguero de los hermanos Torres.

\section{EL CEAD DE MÁLAGA}

El CEAD de Málaga cuenta actualmente con 222 estudiantes que provienen de los veintidós municipios de la Provincia de García Rovira en Santander, y Norte y Gutiérrez en Boyacá; también se atiende a cerca de 80 estudiantes del bachillerato. A la fecha se cuenta con un número de egresados cercano a los 664 profesionales.

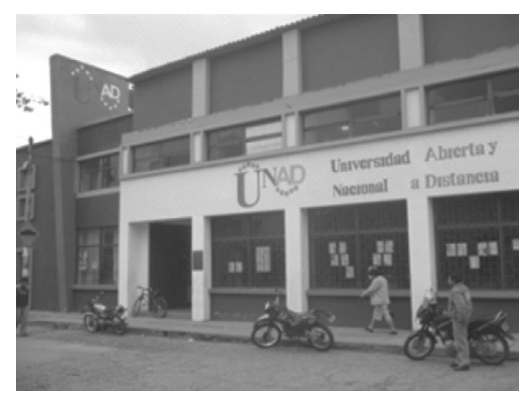

Figura 5. Panorámica del CEAD de Málaga 
La investigación en el CEAD está dinamizada por grupos y semilleros de investigación. Frailejones, del programa de Administración de Empresas, grupo liderado por el docente Germán Garcés, tiene como objeto de investigación lo relacionado con los sistemas de producción agrícola con especial énfasis en el cultivo del tabaco. En cuanto a los semilleros, activos están: Alquimia perteneciente al programa de Regencia en farmacia liderado por la docente Pilar Catherine Márquez Garcés; el semillero Construyendo Futuro pertenece al programa de Administración de Empresas y es liderado por el estudiante Jhonny Pinzón Higuera y apadrinado por la tutora Luz Marina Rueda, y por último, el semillero Somos Humanos del programa de Psicología, es liderado por Geduard Tarazona con el apoyo de la tutora Elizabeth Osorio García.

\section{LA REGIÓN DE PAMPLONA ${ }^{117}$}

Nueva Pamplona del Valle del Espíritu Santo, nombre con que don Pedro de Ursúa y don Ortún Velasco de Velásquez rendían homenaje a la capital de la Provincia de Navarra (España), fue fundada el $1 .^{\circ}$ de noviembre de 1549 .

La oferta ambiental del municipio está fundamentada en las áreas de nacimiento del río Pamplonita, importante fuente hídrica que abastece el acueducto de la capital del departamento Norte de Santander; además, posee algunas áreas de páramo que sustentan el equilibrio hídrico de varios ríos y quebradas que abastecen dentro y fuera del municipio.

La riqueza cultural de Pamplona ha sido enaltecida por notables figuras de renombre nacional e internacional que engrandecen el orgullo del sentir colombiano, entre quienes se encuentran músicos como Oriol Rangel y Bonifacio Bautista, poetas como Jorge Gaitán Durán y Eduardo Cote Lamus y escultores como Eduardo Ramírez Villamizar. La inquietud artística se hace presente en los eventos que a lo largo del año demuestran el interés especial que la pintura, la danza, el teatro, la fotografía y la música despiertan en la juventud pamplonesa.

La producción manufacturera es una actividad económica que representa menos del $10 \%$ de los establecimientos y del empleo de la ciudad. La tercera parte de la industria corresponde a la producción de tejidos de los cuales la mayoría de ellos adelantan sus procesos en forma manual. El segundo renglón es el de producción de alimentos particularmente de panaderías, dulcerías y salsamentarias. Las industrias existentes se caracterizan por su condición microempresarial derivada del poco capital invertido, por su retraso productivo en que no hay mayor división social del trabajo, por el predominio de la producción «artesanal» y por la poca acumulación de capital.

117La información utilizada para la descripción de esta región ha sido tomada del sitio web http://www.pamplonanortedesantander.gov.co/sitio.shtml $\mathrm{apc}=\mathrm{m} 1 \mathrm{~T} 1--\& \mathrm{~m}=\mathrm{f}$ y a partir de la colaboración del docente Carlos Alberto Vera Romero. 


\section{CEAD DE PAMPLONA}

Actualmente se tienen en el CEAD de Pamplona 103 estudiantes matriculados en bachillerato, 271 estudiantes en pregrado y 4 de postgrado, lo que da un total de 378 estudiantes activos en el primer periodo académico del año 2009. El número de egresados supera ya los 490.

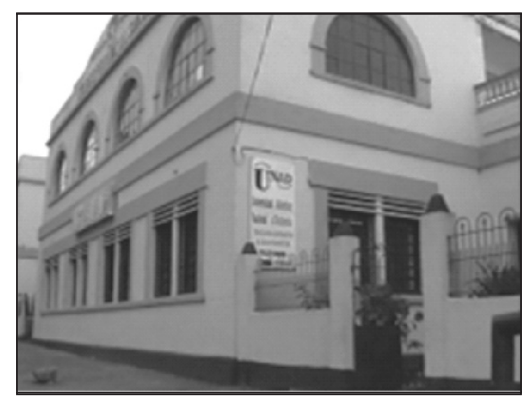

Figura 6. Panorámica del CEAD de Pamplona

En lo que a investigación se refiere la zona cuenta con cuatro semilleros de investigación a saber: el semillero Emprendedores constructores de sueños presente y realidades futuras; el semillero Semillas de esperanza, Conocimiento, esperanza de un mejor futuro; el semillero Sitech, Sistemas tecnológicos al servicio de la humanidad y el semillero Multiplicadores, aprende y decide. La zona cuenta con un grupo en proceso de consolidación denominado GRINDES GRupo de INvestigación para el Desarrollo Económico y Social que apunta a resolver problemas asociados con las líneas: problemas sociales y construcción de intersubjetividades, prospectiva estratégica, salud pública e ingeniería de software.

\section{LA REGIÓN DE VÉLEZ}

Vélez fue la segunda ciudad fundada por la hueste española que incorporó el Nuevo Reino de Granada a la Corona de Castilla; hace parte de la primera ola fundacional de los conquistadores, y su fundación constituye un acto político que significaba el designio «de ocupar la tierra y afirmar el derecho de los conquistadores». El nombre de Vélez fue elegido por Jiménez de Quesada en consulta con los capitanes Gonzalo Suárez Rendón y Martín Galeano, comisionados para realizar la fundación el 3 de julio de 1539.

Con más de cuatro décadas de tradición, el Festival de la guabina y el tiple se proyecta como uno de los más importantes en el país; este evento encierra costumbres propias de los primeros pobladores de la región que se mantienen vivas en la comunidad. De la misma forma, la parranda veleña, hace alarde de las antiguas romerías que se realizaban de un pueblo a otro; también el Desfile de las flores hace parte del ingenio y la creatividad de la gente que elabora diferentes motivos florales. Igualmente, la Feria equina se destaca por la exposición de los mejores ejemplares del país. 


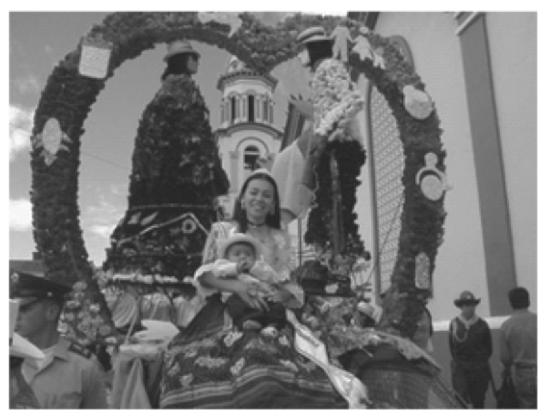

Figura 7. Festividades de Vélez

El municipio de Vélez es el eje central de la economía de la provincia pues hacia allí confluyen las personas de los diferentes municipios a comercializar los productos agrícolas y pecuarios. Es considerado como el principal mercado regional de bovinos, equinos, porcinos y un excelente distribuidor de productos agrícolas. La guayaba es una explotación tradicional asociada a la explotación ganadera, tiene importancia para los ingresos de los campesinos en época de cosecha: octubre, noviembre, diciembre y en mitaca.

El mercado de productos agrícolas se realiza en la plaza de mercado los días sábados; en este intercambio intervienen personas de los diferentes municipios vecinos.

\section{EL CERES DE VÉLEZ}

El 20 de diciembre de 2004 mediante el convenio de cooperación 261 firmado entre la UNAD y el MEN se crea el CERES Vélez con el objetivo de ofrecer programas técnicos, tecnológicos y profesionales a los habitantes del sur del departamento de Santander y del bajo Ricaurte en Boyacá. Actualmente la Universidad Nacional Abierta y a Distancia (UNAD-CEAD) Vélez cuenta con una amplia cobertura y con 153 estudiantes.

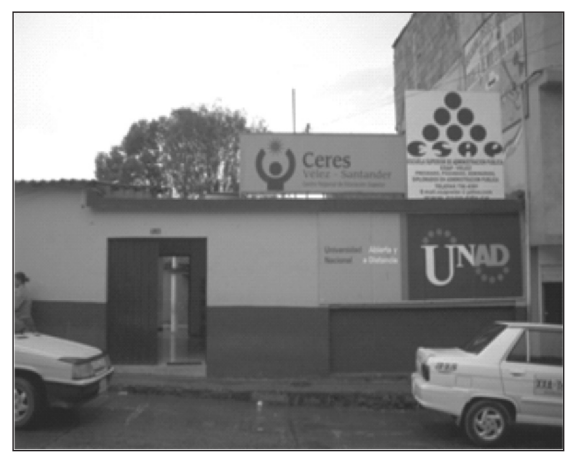

Figura 8. Panorámica del CERES de Vélez 
En este momento cuenta con 83 egresados de los diferentes programas académicos. Los procesos de investigación de la zona están siendo impulsados mediante la implementación de varias estrategias tendientes a la conformación de semilleros de investigación.

\section{LA REGIÓN DE OCAÑA}

La ciudad de Ocaña fue fundada el 14 de diciembre de 1570, por el Capitán Francisco Fernández de Contreras. El nombre de Ocaña le fue puesto al nuevo poblamiento, como homenaje a Fernández del Busto, natural de Ocaña, en España. Los pueblos prehispánicos que habitaron la región, fueron los denominados tradicionalmente como Hacaritamas. ${ }^{118}$

En el transcurso de la guerra de Independencia, la zona de Ocaña cobra singular importancia estratégica. Allí, Bolívar (1813), quien denominó la ciudad «brava y libre», consolidó sus fuerzas para la Campaña Admirable con tropas momposinas y de la región. En 1815, Santander hace su entrada a Ocaña recibiendo allí el nombramiento de Comandante General de las tropas de reconquista del norte.

Entre las principales festividades se cuentan los carnavales ${ }^{119}$ que tienen lugar los días 4, 5 y 6 de enero. Estas festividades populares reemplazaron las antiguas ferias y fiestas a partir del año de 1945; la Semana Santa viene celebrándose desde la época colonial, cuando prelados y autoridades locales presidían las majestuosas procesiones. Este evento fue declarado como Bien de interés cultural de carácter departamental, mediante Decreto 1144 de 31 de diciembre de 2003, expedido por la gobernación de Norte de Santander.

Los aspectos culturales más relevantes giran en torno a las instituciones y grupos independientes, entre ellos: la Corporación Banda Municipal, fundada en 1922; la Academia de Historia de Ocaña que tiene su sede en el Complejo Histórico de la Gran Convención; la Biblioteca Pública Municipal Luis Eduardo Páez Courvel y la Escuela de Bellas Artes Jorge Pacheco Quintero.

\section{CEAD DE OCAÑA}

El CEAD de Ocaña tiene actualmente 377 estudiantes y aproximadamente 75 egresados. En cuanto a investigación está en proceso de consolidación el Grupo de Investigación GEIBO, que entre sus funciones asume el apadrinamiento del semillero Desarrollo y Fortalecimiento de los Servicios Farmacéuticos que se ocupa de estudiar las temáticas relacionadas con el uso de medicamentos en mujeres embarazadas, el uso de anticonceptivos de emergencia y la evaluación de las normas de prescripción de medicamentos; ha estado trabajando también en el diseño e implementación de un centro virtual de información de medicamentos y tóxico.

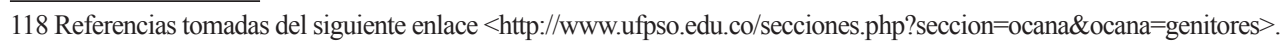

119 Referencias tomadas del siguiente enlace $<\mathrm{http}$ ://joomla.cocota.com/index.php?option=com_content\&task=vie $\mathrm{w} \& \mathrm{id}=56 \&$ Itemid $=89>$. 


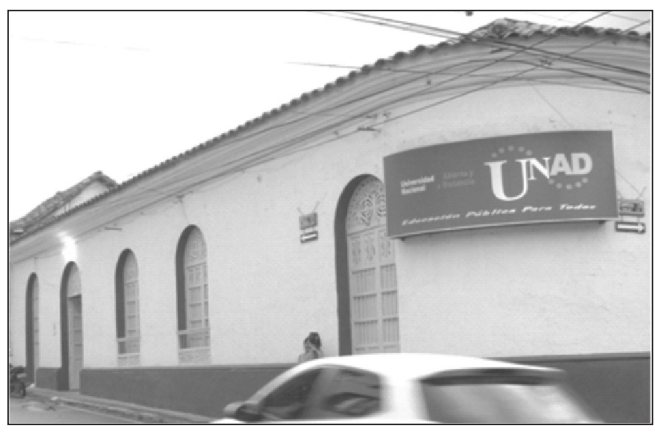

Figura 9. Panorámica del CEAD de Ocaña

El autor desea expresar públicamente agradecimientos a todos los directores de CEAD y CERES de la Zona Centro Oriente y a los docentes y funcionarios del CEAD de Bucaramanga, en especial al profesor Germán Garcés y a Hermes Vargas pues sin su ayuda no hubiera sido posible realizar esta semblanza de la zona.

Y, ya para terminar este recorrido por Centro Oriente hay que decir que se ofrece a nuestros visitantes, además de lo antes señalado, una serie de atractivos turísticos que incluyen los deportes extremos como el canotaje, la espeleología, el rafting, el parapentismo y también el turismo ecológico; además de una excelente oferta gastronómica y de productos artesanales. Entre sus atractivos más importantes se encuentran: Barichara, el pueblito más bello de Colombia, el parque nacional del Chicamocha con el teleférico más grande del mundo, la zona petrolífera de Barrancabermeja y sus alrededores, los festivales de música colombiana de Vélez, entre otros. Se espera que con esta descripción de la zona, los lectores se animen a visitar esta tierra, la de Galán y los comuneros. 